\title{
The Load Distribution of the Main Shaft Bearing Considering Combined Load and Misalignment in a Floating Direct-Drive Wind Turbine
}

\author{
Jingyang Zheng ${ }^{1}$, Jinchen $\mathrm{Ji}^{1}$, Shan $\mathrm{Yin}^{2}$, and Van Canh $\mathrm{Tong}^{3}$ \\ ${ }^{1}$ School of MME, University of Technology Sydney, 2007 Broadway, Sydney, Australia \\ ${ }^{2}$ State Key Laboratory of ADMVB, Hunan University, 410082 Changsha, Hunan, China \\ ${ }^{3}$ Department of UPMS, Korea Institute of Machinery and Materials, 34103 Yuseong-gu, Daejeon, Republic of Korea
}

\begin{abstract}
The main shaft tapered double-inner ring bearing (TDIRB) of floating direct-drive wind turbine system (FDDWT) is one of the most critical components in FDDWT, and its failure accounts for a large proportion of wind turbine malfunctions and faults. Over the past decades, a significant number of methods have been proposed to calculate the contact load distribution along the roller length in TDIRB, since the contact load distribution of roller is the key factor of fatigue life of TDIRB. Most of methods, however, neglected the misalignment of inner ring with respect to outer ring and friction force. In this paper, with the help of comprehensive and accurate quasi-static mathematical method, the contact load distribution of internal loads in TDIRB are analysed by considering the effects of combined loads, angular misalignment and friction force at different wind speeds for FDDWT. The simulation results show that the amount of combined load has an apparent effect on the contact load distribution along the TDIRB raceways and flanges in both rows. Furthermore, the slight change of tilted misalignment has a great influence on the contact load distribution. In addition, the slight angular misalignment easily produces noncontact zone for the bearing raceway in both rows, which is significantly disadvantage for the external load uniform transmitting to each roller.
\end{abstract}

\section{Introduction}

Currently, wind power is one of the cheapest sources for large-scale renewable energy [1]. A historical record of $4,331 \mathrm{MW}$ of new offshore wind power was installed across nine markets globally in 2017 [2]. The generator of FDDWT, unlike traditional geared wind turbine, operates at a varying frequency, directly proportional to the rotor speed. The advantage of this design is the complex high-speed geared drive train common in most conventional wind turbines [3] replaced by a direct-drive generator. Unfortunately, many generators of FDDWT are suffering premature TDIRB failure [4].

The fatigue life of bearing is closely associated with the contact load distribution along the raceways and flanges. Considering misalignment and heavy load conditions, Harris [5] carried out an early study on double-row CRB under radial and thrust load. Puneethkumar M V and Vennapusa V used the FEA and linear method to build up the tapered roller bearing model which were time-consuming and inaccurate $[6,7]$. Van Canh [8] proposed a more accurately mathematical method to calculate the pressure distribution along roller length considering the centrifugal force and gyroscopic moment.

To enhance the modelling level and accuracy with respect to pressure distribution calculation, this paper proposes the model used combination of non-Hertzian elastic half-space theory and slicing technique considering the combined load, angular misalignment and friction force to solve the contact load distribution along the raceways and flanges under the heavy load and low-speed conditions.

\section{Tapered double-inner ring bearing models}

In this section, in order to obtain the contact load distribution and deformation along the roller length, a comprehensive quasi-static model subjected combined load, misalignment and friction is presented here. The typical cross-section construction of an TDIRB is shown in Fig. 1: $\alpha_{i}$ (semi-cone angle of inner raceway), $\alpha_{m}$ (semi contact angle), $\alpha_{o}$ (semi-cone angle of outer raceway), $\varepsilon$ (the semi-cone angle of roller), $l_{w e}$ (effective length of roller), $D_{\max }$ (large end roller diameter), $D_{\min }$ (small end roller diameter), $D_{m}$ (mean roller diameter), $\alpha_{f}$ (inner ring flange angle), $r_{i}$ (semi-minimum inner raceway contact radius), $r_{m}$ (pitch radius) and $r_{o}$ (semimaximum inner raceway contact radius). In order to simplify the analysis, the significant assumptions introduced as follows: the rolling elements are made from purely elastic materials; only elastic deformation 
associated with the local contact area of roller is considered; the outer ring is fixed in space and structural deformation of the bearing components is neglected; the influence of the cages and the roller skewing are also neglected; the components of TDIRB and lubricant keep the same temperature during operation.

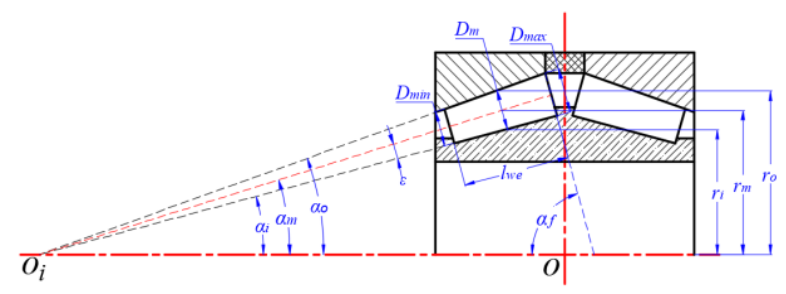

Fig. 1. Basic geometrical dimensions of a TDIRB
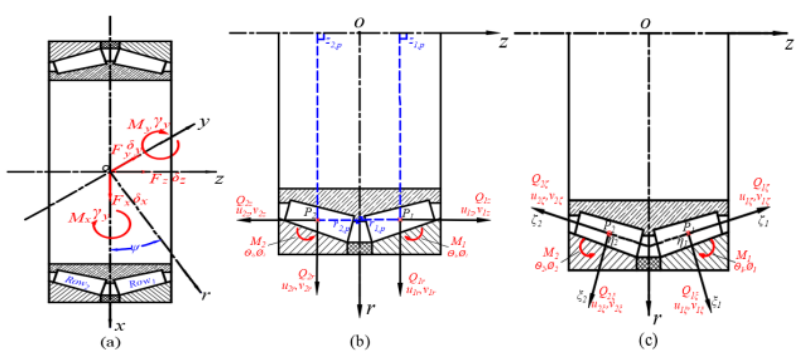

Fig. 2. TDIRB coordinate systems with loads, displacements and angular misalignment: (a) global coordinate system; (b) cylindrical coordinate system; (c) inclined coordinate system.

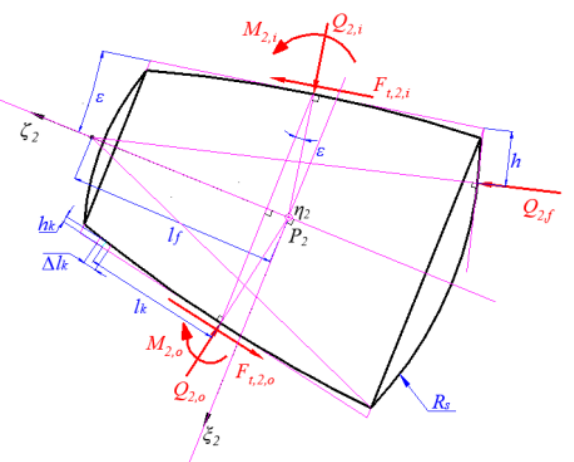

(a)

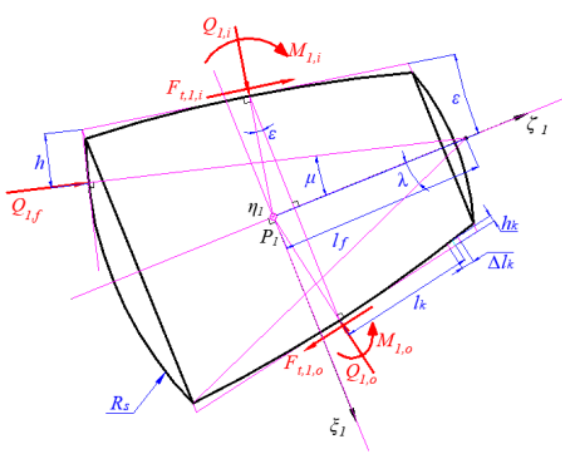

(b)

Fig. 3. Free body diagram of rollers: (a) a roller in row 2; (b) a roller in row 1.

\subsection{Coordinate systems}

The five degree-of-freedom (DOF) TDIRB model is established based on Van Canh's [9] model considering friction force. A right-handed global coordinate system $(x, y, z)$ is introduced with origin located at the centre of TDIRB, where $x$-axis is the vertical axis with positive in a downward direction, $y$-axis is specified at a location angle $\psi=900$ from $x$-axis, and $z$-axis is the TDIRB rotational axis. This global system is fixed with respect to the bearing outer ring, as shown in Fig. 2 (a). Two local cylindrical coordinate systems $\left(r_{M}, \psi, z_{M}\right)$ are also introduced, where the subscript $\mathrm{M}$ denotes the TDIRB row, therefore, $M=1$ refers to row 1 (right row) and $M=2$ refers to row 2 (left row). The reference points of inner ring cross-section are $P 1\left(r_{p}, z_{p}\right)$ and $P 2\left(r_{p},-z_{p}\right)$, as shown in Fig. 2 (b). In addition, two inclined coordinate systems $\left(\xi_{M}, \zeta_{M}, \eta_{M}\right)$ are employed with the inclined angle $\alpha_{m}$ and $-\alpha_{m}$, as shown in Fig. 2 (c). Noted that $\eta_{1^{-}}$ axis is perpendicular to $\left(\xi_{1}, \zeta_{1}\right)$ plane in inward direction, while $\eta_{2}$-axis is normal to $\left(\xi_{2}, \zeta_{2}\right)$ plane in outward direction.

The TDIRB is subjected to an external force vector in the global coordinate system, as shown on Fig. 1,

$$
\{F\}^{T}=\left\{F_{x}, F_{y}, F_{z}, M_{x}, M_{y}\right\}
$$

and the inner ring displacement vector is

$$
\{\delta\}^{T}=\left\{\delta_{x}, \delta_{y}, \delta_{z}, \gamma_{x}, \gamma_{y}\right\}
$$

Meanwhile, the displacement vectors of inner ring cross-section in cylindrical coordinate system and inclined coordinate system are introduced by $\left\{u_{M}\right\}^{T}=\left\{u_{M, r}, u_{M, z}, \theta\right\} \quad$ and $\quad\left\{u_{M, \alpha_{m}}\right\}^{T}=\left\{u_{M, \xi}, u_{M, s}, \theta\right\}$, respectively, where, $\theta$ is the angular displacement of inner raceway cross-section. From the Fig. 2, we can obtain

$$
\begin{gathered}
\left\{u_{M}\right\}=\left[R_{M}\right]\left\{\delta_{M}\right\} \\
\left\{u_{M, \alpha_{m}}\right\}=\left[K_{M}\right]\left\{u_{M}\right\}
\end{gathered}
$$

Similarly, the displacement vectors of rollers in each local coordinate system are described by $\left\{v_{M}\right\}^{T}=\left\{v_{M, r}, v_{M, z}, \phi_{M}\right\} \quad$ and $\quad\left\{v_{M, \alpha_{m}}\right\}^{T}=\left\{v_{M, \xi}, v_{M, \xi}, \phi_{M}\right\}$ respectively, where, $\phi_{M}$ are the angular displacement of rollers in both rows. From Fig. 2, we can calculate

$$
\left\{v_{M, \alpha_{m}}\right\}=\left[K_{M}\right]\left\{v_{M}\right\}
$$

where, $\left[K_{M}\right]$ and $\left[R_{M}\right]$ are the transformation matrices referred to $[8,11]$.

\subsection{Contact deformation}

Fig. 3 shows the free body diagrams of rollers in row 1 and row 2. To commence the analysis, an alternative slicing technique [10] is employed, in which the contact length of roller is divided into ns slice. According to [9, $10]$, the contact deformations $\delta_{M, N, k}$ of the kth laminae in row 1 and row 2 can be calculated by

$$
\delta_{M, N, k}=\delta_{M, N}+\beta_{M, N} \cdot l_{k}-h_{k}
$$

where, $\mathrm{N}$ is the bearing ring index, $N=i$ for inner ring and $N=O$ for outer ring. $\beta_{M, N}$ are the relative angular misalignment between rollers and inner ring, as well as rollers and outer ring respectively calculated by

$$
\beta_{M, i}=\theta-\phi_{M}
$$




$$
\beta_{M, o}=\phi_{M}
$$

$l_{k}$ is the axial position of lamina $k$, which ranges from $-l_{w e} / 2$ to $l_{w e} / 2$, as shown in Fig. $3 . h_{k}$ is the crown drop at lamina $k$ caused by the modified roller profile. According to $[8,11,13]$, we employ the fullylogarithmic roller profile and flat raceway profiles. The mathematical function of roller profile adopted for TRB was given by in reference [12]. $\delta_{M, N}$ are the contact deformations between rollers and rings caused by their translation motion. $\delta_{M, f}$ are the contact deformation between big-end of rollers and inner ring flange. $\delta_{M, N}$ and $\delta_{M, f}$ are presented by, respectively $[9,10]$,

$$
\begin{gathered}
\delta_{M, i}=\left(u_{M, \zeta}-v_{M, \zeta}\right) \cdot \cos \varepsilon-\left(u_{M, \zeta}-v_{M, \zeta}\right) \cdot \sin \varepsilon \\
\delta_{M, o}=v_{M, \zeta} \cdot \cos \varepsilon+v_{M, \zeta} \cdot \sin \varepsilon \\
\delta_{M, f}=\left(u_{M, \zeta}-v_{M, \zeta}\right) \cdot \sin \mu_{0}+\left(u_{M, \zeta}-v_{M, \zeta}\right) \cdot \cos \mu_{0}+ \\
l_{f} \cdot\left[\cos \mu_{0}-\cos \left(\mu_{0}+\beta_{M, i}\right)\right]
\end{gathered}
$$

where, $\mu_{0}$ is the angle between roller centre line and roller-flange contact line, $l_{f}$ is the distance between reference points $P 1$ and $P 2$ and the intersection of the flange force and the centre line of roller, as shown in Fig. 2 and Fig. 3.

\subsection{Internal load and friction}

As aforementioned, the alternative slicing technique is used for the non-Hertzian line contacts, and the contact forces $q_{M, N, k}$ at the $k$ th lamina are calculated by the following equations

$$
q_{M, N, k}=c \cdot \delta_{M, N, k}^{10 / 9} \cdot \Delta l_{k} ; \quad \delta_{M, N, k}>0
$$

where, $\Delta l_{k}$ is the width of a lamina. The contact stiffness $c$ is a constant, which depends on the material properties and contact geometry at the roller and raceway [5]. Therefore, the total contact loads $Q_{M, N}$ and moments $M_{M, N}$ along the roller length can be obtained

$$
\begin{gathered}
Q_{M, N}=\sum_{k=1}^{n_{S}} q_{M, N, k} \\
M_{M, N}=\sum_{k=1}^{n_{S}} q_{M, N, k} \cdot l_{k}
\end{gathered}
$$

In terms of the contact force between big roller-end and inner raceway flange, $Q_{M, f}$ are regarded as classical Hertzian force and conveniently calculated as follows

$$
Q_{M, f}=c_{f} \cdot \delta_{M, f}^{3 / 2} ; \quad\left(\delta_{M, f}>0\right)
$$

where, the contact stiffness $c_{f}$ is a constant, which depends on the material properties and radius of the bigend spherical shape of rollers [5].

Since the main shaft TDIRB in FDDWT running with low speed and heavy load condition, the effects of centrifugal and gyroscopic forces are considered insignificant, while the thrust frictional force in the roller-raceway interface is relatively important for the static equilibrium in $\left(\xi_{M}, \zeta_{M}\right)$ planes $[11,12]$, see Fig. 3 . The friction force is simply described by

$$
F_{t_{M, N}}=Q_{M, N} \cdot \mu_{z, \max }
$$

where, $\mu_{z, \max }$ is the maximum of the traction coefficient in the axial direction.

\section{Tapered double-inner ring bearing equilibirum}

The quasi-static analysis model of TDIRB are described based on the equilibrium of all rollers and inner ring. In the following section, the modelling process and solution approach are presented for the aforementioned equilibrium equations.

\subsection{Quasi-static equilibrium equations of rollers}

Based on the free body diagrams and in [8], the rollers equilibrium equations can be calculated by

$$
\left\{F_{M, r}\right\}=\left\{\begin{array}{l}
F_{M, \xi} \\
F_{M, \zeta} \\
M_{M, \eta}
\end{array}\right\}=\left\{\begin{array}{l}
Q_{M, i} \cos \varepsilon-Q_{M, o} \cos \varepsilon+Q_{M, f} \sin \mu_{0}+\left(F_{t_{M, i}}+F_{t_{M, o}}\right) \sin \varepsilon \\
-Q_{M, i} \sin \varepsilon-Q_{M, o} \sin \varepsilon+Q_{M, f} \cos \mu_{0}+\left(F_{t_{M, i}}-F_{t_{M, o}}\right) \cos \varepsilon \\
M_{M, i}-M_{M, o}+Q_{M, f} l_{f} \sin \mu_{M}+0.5\left(F_{t_{M, i}}+F_{t_{M, o}}\right) D_{m} / \cos \varepsilon
\end{array}\right\}=\left\{\begin{array}{l}
0 \\
0 \\
0
\end{array}\right\}
$$

where, $\mu_{M}$ are the angles between inner ring flange contact line and roller centre line for both rows as the shift of the contact point on both inner raceway flange and roller big end under load. In equation (17), the nominal angle $\mu_{0}$ is used for the force equations as the effect of its changes under load is very small. For moment equations, however, the actual value is considered since it is important for line contact rollers.

To solve the set of nonlinear equations (17), the iterative Newton-Raphson method is employed with Jacobian matrices $\left[J_{M, r}\right]$ and increments $\left\{\Delta_{v_{M, \alpha_{m}}}\right\}$, as follow

$$
\left\{F_{M, r}\right\}+\left[J_{M, r}\right]\left\{\Delta v_{M, \alpha_{m}}\right\}=\{0\}
$$

\subsection{Quasi-static equilibrium equations of inner ring}

To the determine the equilibrium equations of rollers, the inner ring contact load vectors in inclined coordinate system $\left(\xi_{M}, \zeta_{M}, \eta_{M}\right)$ can be presented by

$$
\left\{Q_{M, \alpha_{m}}\right\}=\left\{\begin{array}{l}
Q_{M, \xi} \\
Q_{M, \zeta} \\
T_{M, \eta}
\end{array}\right\}=\left\{\begin{array}{c}
-Q_{M, i} \cos \varepsilon-Q_{M, f} \sin \mu_{0}-F_{t_{M, i}} \sin \varepsilon \\
Q_{M, i} \sin \varepsilon-Q_{M, f} \cos \mu_{0}-F_{t_{M, i}} \cos \varepsilon \\
-M_{M, i}-Q_{M, f} l_{f} \sin \mu_{M}-0.5 F_{t_{M, i}} D_{m} / \cos \varepsilon
\end{array}\right\}
$$

Subsequently, the correspondent contact load vectors in cylindrical coordinate systems can be found using transformation matrix $\left[K_{M}\right]$, by

$$
\left\{Q_{M}\right\}=\left[K_{M}\right]^{T}\left\{Q_{M, \alpha_{m}}\right\}
$$

Then, the contact load vectors can be transformed to the global coordinate system using the transformation matrix, resulting in equivalent load vectors as follows

$$
\left\{f_{M}\right\}=\left[R_{M}\right]^{T}\left\{Q_{M}\right\}
$$

Finally, the equilibrium equations of the inner ring in global coordinate system are presented with combining 
all the aforementioned equivalent loads and external loads as

$$
\left\{F_{g}\right\}=\{F\}+\sum_{j=1}^{Z}\left\{f_{M}\right\}_{j}=\{0\}
$$

where, $Z$ is the number of rollers in each row. Similar to the rollers equations, the set of non-linear equations (22) are then solved by the numerical Newton-Raphson iterative scheme.

Table 1. TDIRB geometrical and material properties.

\begin{tabular}{|l|l|}
\hline$Z$-Number of rollers in each row & 40 \\
\hline$\alpha_{i}$-Semi-cone angle of inner raceways (degree) & 10.8 \\
\hline$\alpha_{o}$-Semi-cone angle of outer raceways (degree) & 12.5 \\
\hline$\varepsilon$-Semi-cone angle of roller (degree) & 0.85 \\
\hline$l_{\text {we }}$-Effective contact length of tapered roller $(\mathrm{mm})$ & 158.75 \\
\hline$D_{\text {max }}$-Large end roller diameter $(\mathrm{mm})$ & 83.44 \\
\hline$D_{\text {min }}$-Small end roller diameter $(\mathrm{mm})$ & 78.49 \\
\hline$d_{m a x}$-Maximum inner raceway contact diameter $(\mathrm{mm})$ & $1,055.15$ \\
\hline$d_{m i n}$-Minimum inner raceway contact diameter $(\mathrm{mm})$ & 994.95 \\
\hline$E$-Elastic modulus $(\mathrm{GPa})$ & 208 \\
\hline$v$-Poisson ratio & 0.3 \\
\hline
\end{tabular}

\subsection{Numerical analysis}

The analysis of the axial force and misalignment influence of inner ring on the internal load between rollers and raceways is conducted on a TDIRBLM287649D [15] used in a 5 MW FDDWT and manufactured by Timken. The bearing geometrical and material properties are presented in Table 1. The rated generator speed of the $5 \mathrm{MW}$ FDDWT is $12.1 \mathrm{rpm}$. The external load, $F_{x}$, applied on generator shaft along $x$-axis onto the inner ring is $1090 \mathrm{KN}$. A zero axial preload is employed for the purpose of simulation. In realistic operation conditions, certain preload magnitude can be applied on TDIRB.

First, as the short-term wind speed increased by 1 $\mathrm{m} / \mathrm{s}$ per 10 minutes from $4 \mathrm{~m} / \mathrm{s}$ to $25 \mathrm{~m} / \mathrm{s}$, hence, the axial force is assumed to increase linearly from $350 \mathrm{KN}$ to 650 $K N$ and the misalignment angle, $\gamma_{y}$, is $0 \mathrm{mrad}$. The results of the quasi-static model consist of series of simulations carried out by Matlab, as shown in Fig. 4. The comparisons in Fig. 4 show that the contact loads between roller and inner ring are similar to those between rollers and outer ring in both rows. Then, the contact loads of row 1 are obviously bigger than those in row 2. A similar situation occurs for the roller and flange contact load distribution. Furthermore, the maximum contact loads between rollers and raceways as well as roller end and flange occur at the roller location angle $\psi=0^{\circ}$ (bottom of the TDIRB), while the minimum contact loads occur at the roller location angle $\psi=180^{\circ}$ (top of the TDIRB). Obviously, the number of roller that comes into contact with raceways gradually increases around the top of row 1, while it reduces around the same location angle of row 2. The roller to inner and outer raceways contact loads at the azimuth angle $\psi=0^{\circ}$ in row 1 experience obvious increasing from $89.98 \mathrm{KN}$ to $108.2 \mathrm{KN}$, whereas the contact loads in the same location in row 2 show an apparent decrease form 34.84
$K N$ to $9.148 K N$. Similarly, the roller end-flange contact loads increase from $2.769 K N$ to $3.331 K N$ for the rollers in bottom of row 1 and reduce from $1.072 K N$ to 0.2816 $K N$ for the rollers in the same position of row 2. In comparison to the results presented in Fig. 1 and Fig. 2 of [16] and Fig. 11 of [13], the aforementioned results are in good agreement, and the presented numerical quasi-static model follows the same trends as in [13] and [16]. In summary, this model shows a good agreement with the reference model.
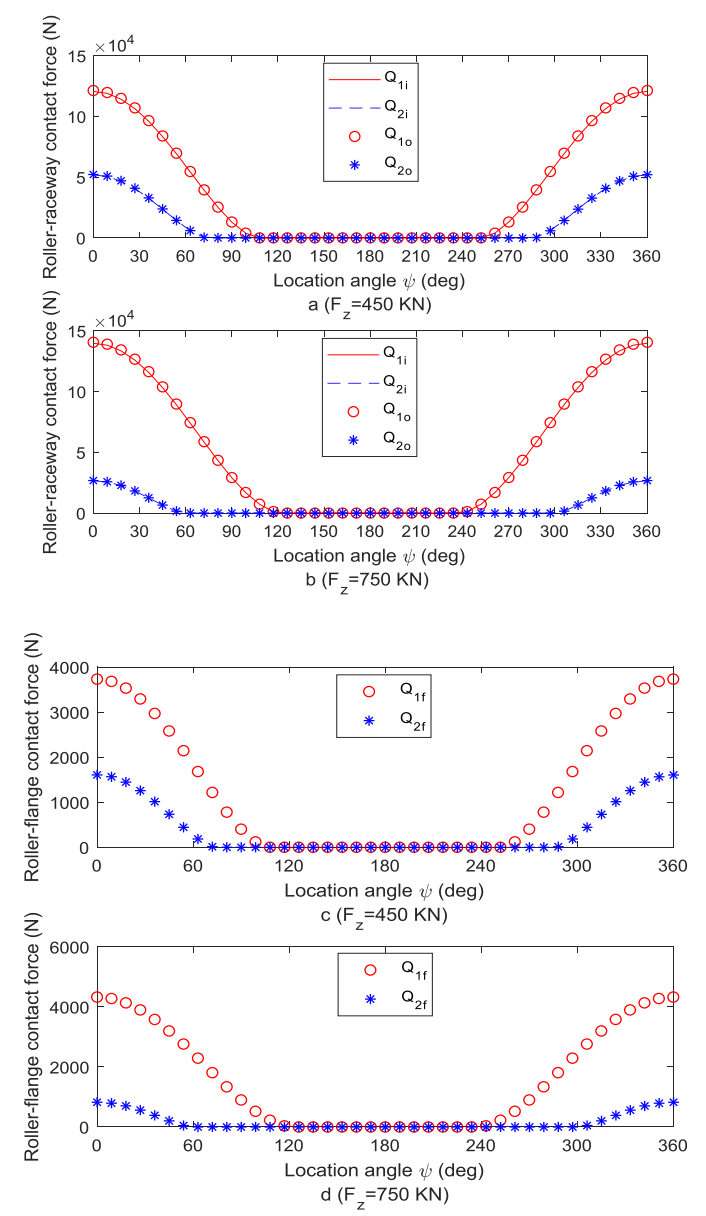

Fig. 4. Contact forces distribution under different axial forces.

Second, to investigate the influence of misalignment on the contact load distribution, we specify the misalignment angle ranging from $1 \mathrm{mrad}$ to $4 \mathrm{mrad}$ with the constant radial and axial forces, $F_{x}=1090 \mathrm{KN}$ and $F_{z}=550 \mathrm{KN}$ respectively, and the results are shown in Fig. 5. A slight increase of the angular misalignment produces a significant change on the contact load distribution of rollers. It is found from Fig. 5 (a) to Fig. 5 (d) that at the bottom of bearing, the roller-raceway contact loads increase from $46.21 \mathrm{KN}$ to $140 \mathrm{KN}$ for row 1 and from $233.71 K N$ to $281.6 K N$ for row 2, respectively, with the misalignment angle growing from $1 \mathrm{mrad}$ to $4 \mathrm{mrad}$. Moreover, the contact loads in row 2 are much larger than those in row 1. Similarly, at the location of $\psi=180^{\circ}$, the contact loads show an obvious increase from $11.85 \mathrm{KN}$ and $0 \mathrm{KN}$ to $249.8 \mathrm{KN}$ and 40.49 $K N$. Meanwhile, there occurs noncontact zone in inner raceway and the corresponding outer raceway, which 
decrease by more than $50 \%$ with the increase of angular misalignment. At the location around $\psi=90^{\circ}$ and $\psi=270^{\circ}$, the contact loads show the same decline trend from $26.57 \mathrm{KN}$ to $15.93 \mathrm{KN}$ in row 1 , whereas the noncontact exists in row 2. Compared with the contact loads along the raceways, the roller-flange contact loads experience the same trend as shown in Fig. 5(c) and 5(d), and the maximum loads come up to $7.688 \mathrm{KN}$ at the top of bearing in row 1 and $8.667 \mathrm{KN}$ at the bottom of bearing in row 2 .
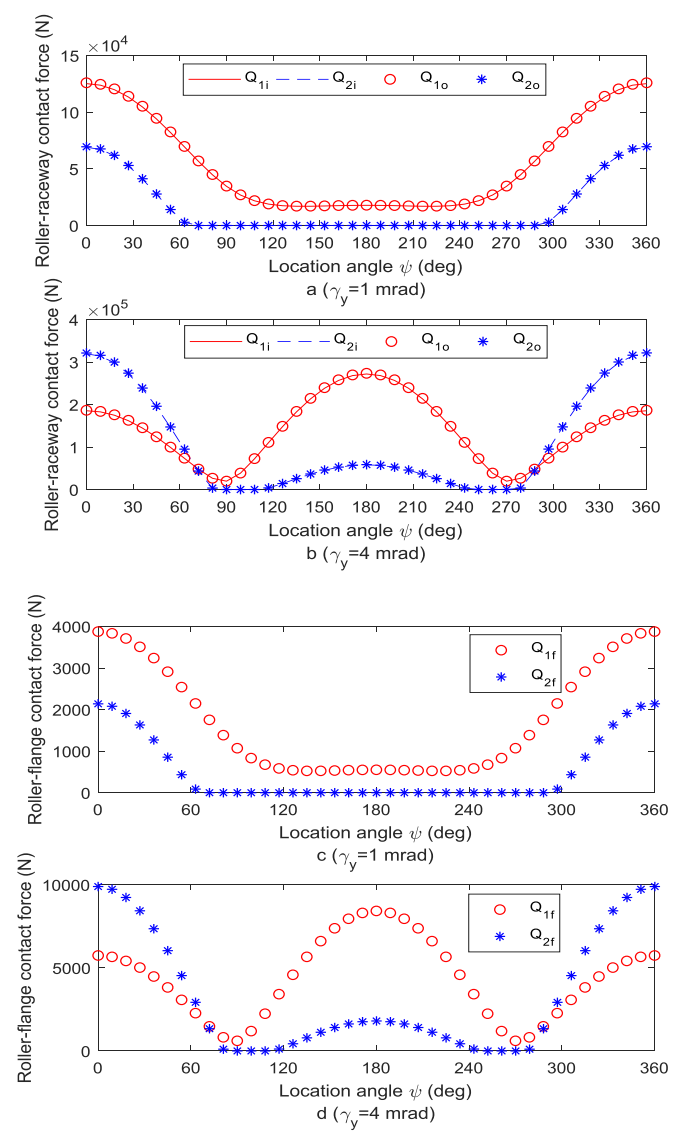

Fig. 5. Contact forces distribution under different angular misalignments.

\section{Conclusions}

This paper puts forward a comprehensive quasi-static model that utilizes the precise alternative slicing technique and non-Hertzian contact estimation to accurately simulate the contact load distribution along the raceway and flange, especially for heavy load and low speed operating conditions. The detailed contact load results are obtained via simulation, which take into account the influence of combined loading and tilted misalignment between the raceways. The simulation results show that the amount of combined load has an apparent effect on the contact load distribution along the TDIRB raceways and flanges in both rows.

Furthermore, the slight change in tilted misalignment has a great influence on the contact load distribution. The variation of contact loads distribution in row 1 and 2 show a contrary tendency. With the increasing of misalignment angle, the contact loads of rollers around the bottom of TDIRB in row 1 and the top of TDIRB in row 2 increase significantly. In addition, a slight angular misalignment produces noncontact zone for the bearing raceway in both rows, which is significantly disadvantage for the external load uniform transmitting to each roller. These aforementioned results also potentially presented the importance of combined load and misalignment influences analysis for the pressure distribution of rollers and fatigue life of TDIRBs in the following work.

\section{Acknowledgement}

The author thanks the University of Technology Sydney and Chinese Scholarship Council for funding the research work.

\section{References}

1. CEC. Wind energy. Clean Energy Council; 2018.

2. GWEC Global Wind 2017 Report - A Snapshot of Top Wind Markets in 2017: Offshore Wind. 2017

3. Zhao M, Ji JC. Nonlinear torsional vibrations of a wind turbine gearbox. Applied Mathematical Modelling. 2015; 39:4928-50.

4. Matthew W, Jon T, Won S, Peter T. Improving wind turbine drivetrain bearing reliability through pre-misalignment. Wind Energy. 2014; 17:1217-30.

5. Roller Cpot. Analysis of contact pressure distribution of the straight and crowning profiles of tapered roller bearing. 2014.

6. Sivareddy VV. Modelling and analysis of deformation on a flexure bearing in linear compressor. 2014.

7. Harris TA, Kotzalas MN. Advanced concepts of bearing technology: rolling bearing analysis: CRC Press; 2006.

8. Tong V-C, Hong S-W. Characteristics of tapered roller bearing subjected to combined radial and moment loads. International Journal of Precision Engineering and Manufacturing-Green Technology. 2014; 1:323-8.

9. Tong V-C, Hong SW. Effects of Roller Profile on the Stiffness of Tapered Roller Bearings. Journal of Automation and Control Engineering. 2015; 3:151-6.

10. Andréason S. Load distribution in a taper roller bearing arrangement considering misalignment. Tribology. 1973; 6:84-92.

11. Warda B, Chudzik A. Effect of ring misalignment on the fatigue life of the radial cylindrical roller bearing. International Journal of Mechanical Sciences. 2016;111-112:1-11.

12. ISO/TS-16281. Rolling bearings-Methods for calculating the modified reference rating life for universally loaded bearings. 2008 . 
13. Nelias D, Bercea I, Paleu V. Prediction of Roller Skewing in Tapered Roller Bearings. Tribology Transactions. 2008; 51:128-39.

14. Oh KP. Analysis of a Needle Bearing. Journal of Tribology. 1984; 106:78-87.
15. Timken Tapered Roller Bearing Catalog. 2018.

16. Nélias D, Bercea I, Mitu N. Analysis of DoubleRow Tapered Roller Bearings, Part II - Results: Prediction of Fatigue Life and Heat Dissipation. Tribology Transactions. 2003; 46:240-7. 